\title{
Tracking and vertexing in Belle II
}

\author{
Bianca Scavino* ${ }^{*}$ on behalf of the Belle II tracking group \\ Johannes Gutenberg Univ. of Mainz, Mainz, Germany \\ E-mail: bscavino@uni-mainz.de
}

Belle II is a next generation B-factory experiment at the SuperKEKB collider. In early 2019 the fully operational detector will start taking data. The goal is to collect a statistics 50 times larger than the one collected by its predecessor Belle, namely an integrated luminosity of $50 \mathrm{ab}^{-1}$. The Belle II tracking detectors are designed to reconstruct charged particles trajectories whose momentum can be as small as $50 \mathrm{MeV} / \mathrm{c}$ with excellent resolution on both impact parameters and momentum. In this contribution, the tracking and vertexing algorithms implemented in the Belle II software framework are presented, together with the performances on simulated $\Upsilon(4 S) \rightarrow B \bar{B}$ events and on data collected during the detector commissioning phase.

The 27th International Workshop on Vertex Detectors - VERTEX2018

22-26 October 2018

MGM Beach Resorts, Muttukadu, Chennai, India

\footnotetext{
*Speaker.

${ }^{\dagger}$ This work was supported by the Bundesministerium für Bildung und Forschung under Grant No. 05H15UMKBA.
} 


\section{The challenges of tracking at Belle II}

Belle II is a multipurpose detector operated at the SuperKEKB asymmetric collider in Tsukuba, Japan. SuperKEKB is a major upgrade of the KEKB accelerator, used from 1999 to 2010 in the Belle experiment. The facility is designed to collide electrons and positrons at a center of mass energy of $\sqrt{s} \sim 10 \mathrm{GeV} / \mathrm{c}^{2}$, that corresponds to the region of the $\Upsilon$ resonances. Most of the data will be collected while operating on the $\Upsilon(4 S)$ peak of the cross section, which is just above the threshold to produce one pair of $B$-mesons. To study the time-dependent $\mathrm{CP}$ asymmetries, a fundamental requirement to fulfill is a good spatial resolution to resolve the two decay vertices of the $B$-mesons coming from the $\Upsilon(4 S)$. One difference of the accelerator with respect to its predecessor is the sizeably reduced boost of the center of mass frame. This allows to increase instantaneous luminosity and increases detector hermeticity, but it has a drawback in reducing the flight length of the $B$-mesons, which translates into the requirement of a vertex resolution improved by a factor of 2. With regard to the event topology, in one $\Upsilon(4 S)$ event there are on average 11 charged tracks. Tab. 1 shows the average fraction of charged particles produced according to the type. As it can be seen from the numbers, pions are the largest fraction. Another feature of the event topology is the presence of many soft tracks: most of the particles have momenta well below $1 \mathrm{Gev} / \mathrm{c}$. This is shown in Fig. 1, that represents the normalized distribution of the momentum $(p)$ for the 5 different types of charged particles.

The experiment peak luminosity goal is $8 \times 10^{35} \mathrm{~cm}^{-2} \mathrm{~s}^{-1}, 40$ times higher than the peak luminosity reached by KEKB. The detector occupancy stemming from machine background (particles lost by beam gas and Touschek scattering and particles lost by non linearities in the machine lattice) is expected to be very high as a consequence of the high beam currents, small emittances and large beam tune shifts needed to reach the design luminosity. Moreover, the electromagnetic processes occurring at the interaction point, radiative Bhabha and electron positron pair production (whose cross sections is order of several mbarn) are going to dominate the beam particle loss rate at nominal luminosity. The number of background hits exceeds the signal hits by two orders of magnitude, and this poses a very challenging task for the tracking detectors and for the software reconstruction. Despite the structure of the Belle II detector remaining similar to its predecessor Belle, because of the upgrade of the accelerator, a major upgrade was necessary, as well. In its new design, Belle II is composed of a PiXel vertex Detector (PXD), a Silicon Vertex Detector (SVD), a Central Drift Chamber (CDC), a Time-Of-Propagation (TOP) counter, a proximity-focusing Aerogel Ring-Imaging CHerenkov (ARICH), an Electromagnetic CaLorimeter (ECL) and a $K_{L}$ and

\begin{tabular}{|c|c|c|c|c|c|}
\hline Particle type & $\pi$ & $K$ & $e$ & $\mu$ & $p$ \\
\hline Avg. fraction & $73.4 \%$ & $14.3 \%$ & $5.9 \%$ & $4.4 \%$ & $2.0 \%$ \\
\hline
\end{tabular}

Table 1: Fraction of particles produced according to the type in a generic $\Upsilon(4 S)$ event. The numbers were obtained using a Monte Carlo simulation, generating 10000 events using the EvtGen package [1]. 


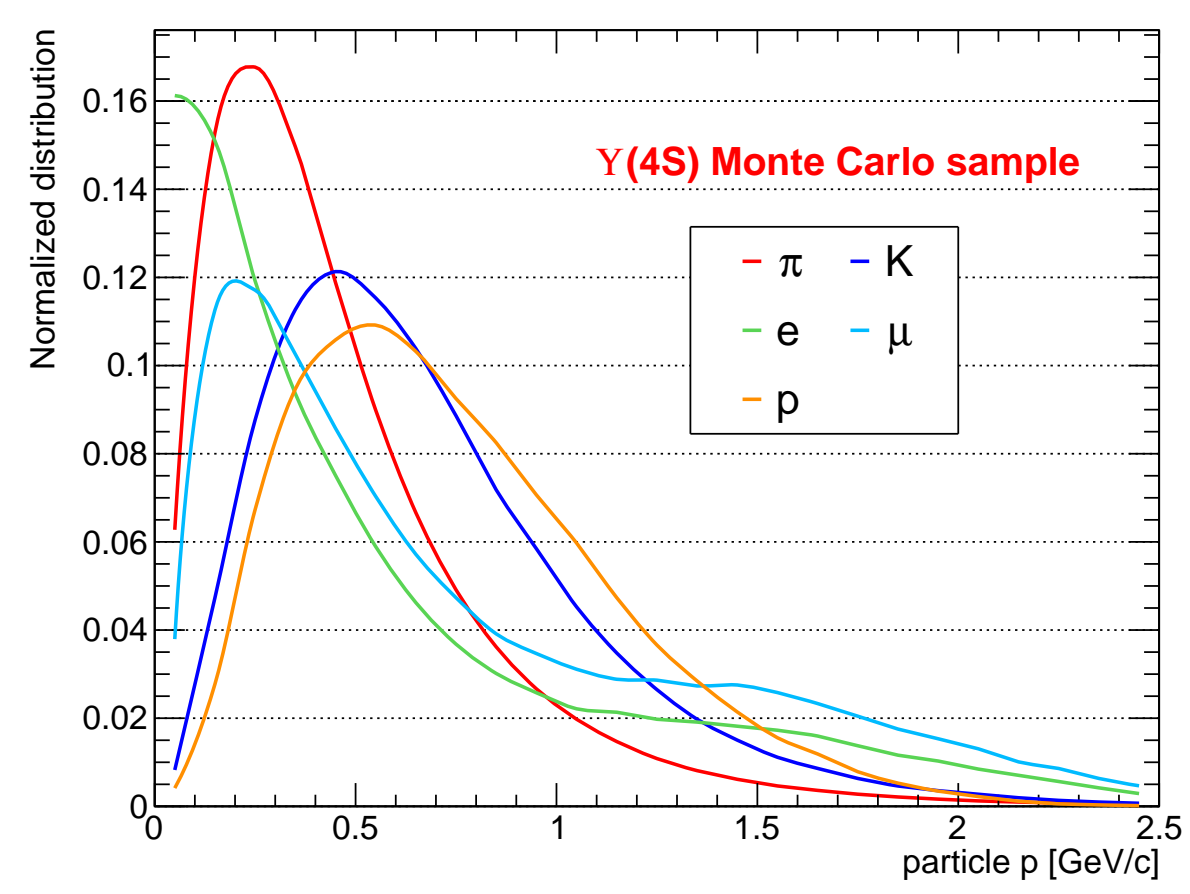

Figure 1: Normalized momentum distributions for 5 different types of particles. The plot was obtained using a Monte Carlo simulation, generating 10000 generic $\Upsilon(4 S)$ events with the EvtGen package [1].

Muon detector (KLM). A schematic view of the detector is shown in Fig. 2, and a more exhaustive description of all the subsystems can be found in [4].

\section{Track Finding}

In its current conception, the workflow starts from a CDC standalone track finding. The outcome of this first step is a collection of CDC tracks that are used as seeds to whom SVD hits are attached using a Combinatorial Kalman Filter (CKF). The SVD hits left not attached are used for a SVD standalone track finding. After merging the two lists obtained, as a last step in the chain the PXD hits are added to the track candidates using again a CKF. By design the whole tracking data-flow can be easily modified allowing to plug in the reconstruction sequence additional track finding tools with a minimum amount of work.

\subsection{CDC track finding}

The CDC track finding combines different algorithms having in common the same starting point: a collection of CDC hits. The very first step implemented is a background filter based on a FastBDT (Fast Boosted Decision Tree), a speed-optimized multivariate classification algorithm developed for the Belle II experiment [5]. For the background rejection, the classification is based on 


\section{Belle II Detector}

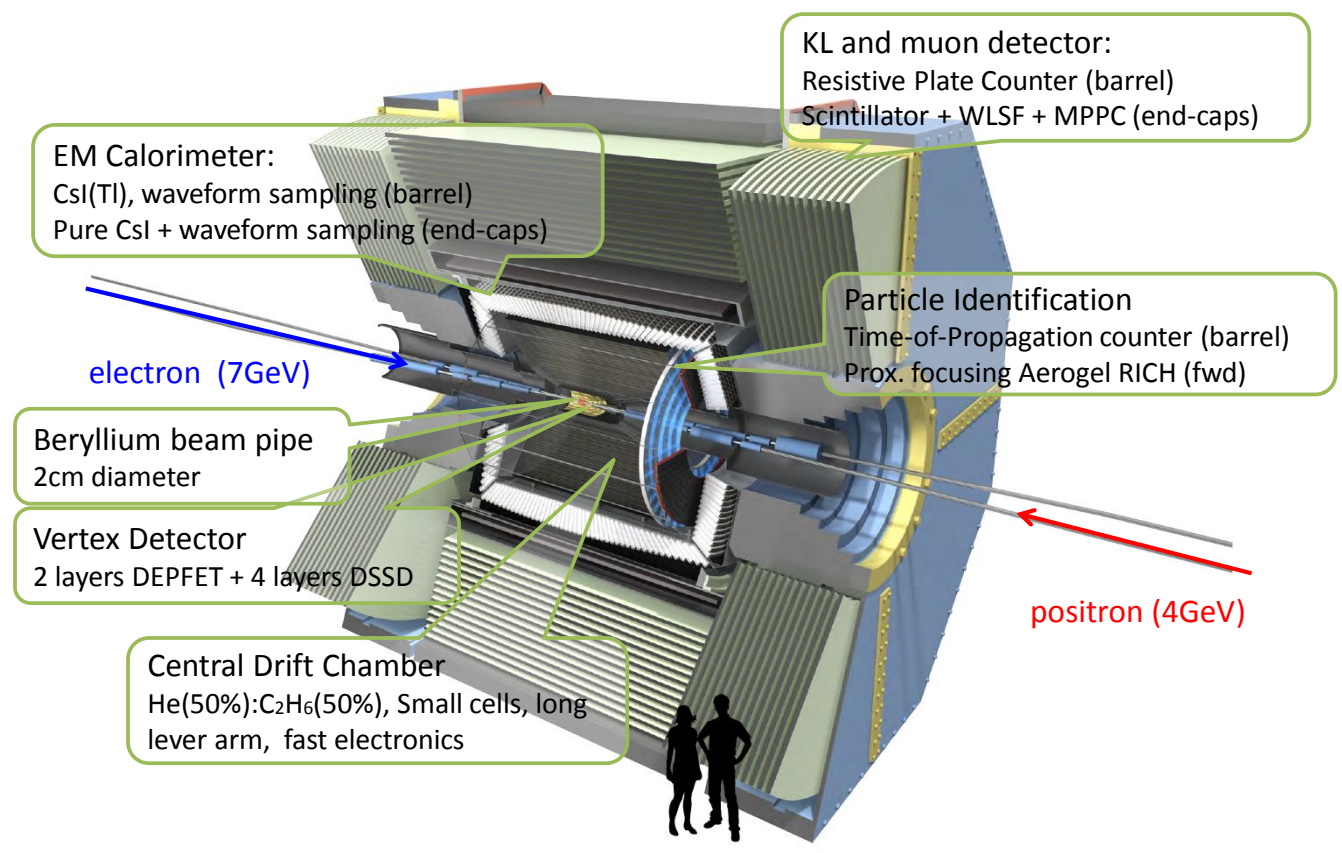

Figure 2: 3D view of the Belle II detector [3].

clustered hits shape variables. In its first implementation the optimization is based on Monte Carlo simulation, but the plan is to tune it using single beam data when available. After this, two track finding algorithms are implemented on the same set of CDC hits: a global one based on Legendre transformation, and a local one based on a cellular automaton $[6,7]$.

The idea behind the global algorithm is to transform the drift circles using Legendre transformation and to map the hits onto the Legendre plane. Every hit in the CDC can be geometrically represented as a circle, having the fired sense wire as center and the hit drift length as radius. At the first stage, considering only the $x y$ projection of the CDC hits, particle trajectories are reconstructed as $2 \mathrm{D}$ circles. In a conformal mapping, all circles passing through the origin of the mapping are transformed into straight lines. Thus particle trajectories coming from the interaction point become straight lines. Furthermore, in the conformal mapped space it is still true that a particle trajectory is always tangent to the drift circle induced. In the end, the first step of the Legendre-based track finding is designed to find common tangents to a set of conformally mapped drift circles. Moving forward, it is possible to reduce the problem of the Legendre-based pattern recognition to the problem of the identification of the most populated regions in the Legendre space. This is carried out using a 2-dimensional binary search towards the possible track candidates.

In parallel to the global tracking algorithm just described, a local one based on a Cellular Automaton technique is implemented, employed on graphs of vertices connected by edges. This operates without any assumption on the origin of the tracks, therefore it is a useful approach to detect displaced tracks. The basic idea is to search for connected hits (so called segments) in the CDC 
superlayers. The whole process is divided into two consecutive stages:

- Segment building stage: vertices formation by combining 3 neighboring hits and assuming a unique trajectory passing through them (left-right passage hypothesis); linear trajectory extraction; edges creation from neighboring triplets sharing two hits;

- Track building stage: combination of individual segments to longer tracks.

The two track finding algorithms are applied on the whole hits set. The results of both approaches are then combined, using the collection of track candidates obtained from the global method as a baseline. A multivariate approach based on FastBDT [5], and trained using simulated events, is used to add segments from the local finder to the global track candidates.

\subsection{SVD track finding}

The starting point of the SVD track finding is a collection of SVD hits. These are at first fed to a CKF, that tries to attach them to the existing CDC track candidates using the principles of the Kalman Filter for track finding [8,9]. All the hits which remain unutilized are used as input for the SVD standalone track finding algorithm, after being converted to space points (a detector-independent format). These space points are then sorted into a sector map, used for filtering possible hit combinations. Here, a few important concepts have to be introduced:

- Space point: global 3-dimensional coordinates of a hit in the SVD and PXD;

- Segment: combination of two space points;

- Sector: virtual subdivision of the SVD sensors. Typically, the number of subdivisions per sensor is 9;

- Friend sectors: different sectors crossed by the same particle. The friendship relations are established using Monte Carlo simulation, during the sector maps training;

- Sector map: network of sectors, where each sector is connected to its own friends.

Segments are searched only on friend sectors, which highly reduces the number of combinations, and the resulting compatible combinations are then used for a Cellular Automaton algorithm. What comes out from this is a set of track candidates with potential overlaps, therefore a Greedy neural network is applied. This finally results in a unique collection of SVD track candidates.

As a last step of the track finding chain, the two collections of CDC and SVD track candidates are merged into one, and finally the PXD hits are attached with a CKF. The reason not to include PXD hits in a global standalone vertex finder, and to add them in a second moment instead, is related to the nature of the PXD itself: because of its proximity to the beam pipe and to the low read out frequency of its sensors, it is subject to a very high beam background induced hits rate. Omitting them from the track finding algorithm simplifies by far the combinatorial problem. Moreover, the option to include them from the beginning is implemented in the algorithm, and it is possible to switch it on in any moment, if desired. 


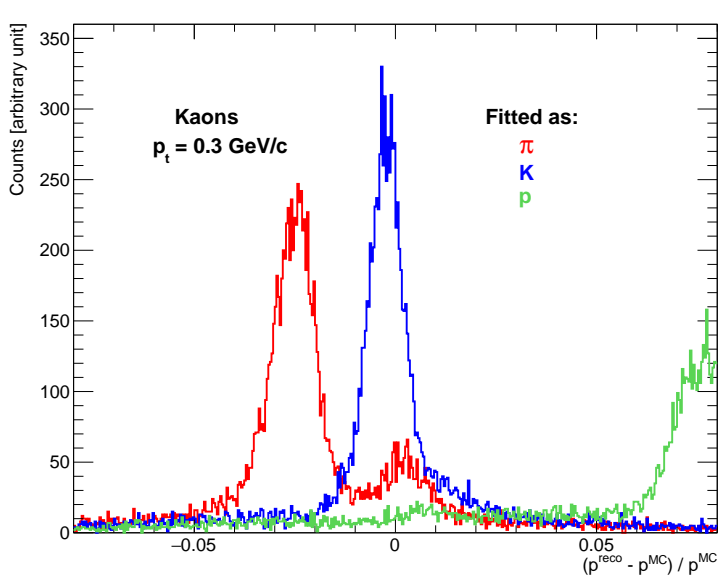

(a) Kaon Monte Carlo sample with fixed transverse momentum $p_{t}=0.3 \mathrm{GeV} / c$

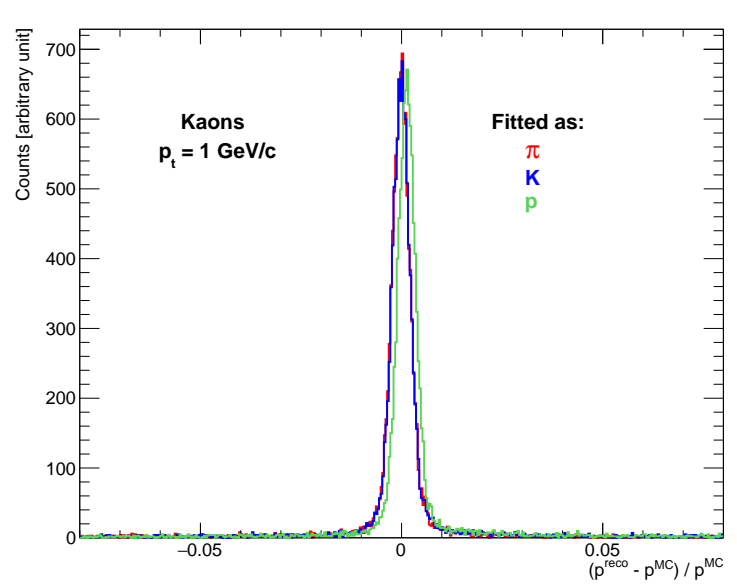

(b) Kaon Monte Carlo sample with fixed transverse momentum $p_{t}=1.0 \mathrm{GeV} / \mathrm{c}$

Figure 3: Momentum resolution of a kaon Monte Carlo sample generated at a fixed polar angle $\left(\theta=60^{\circ}\right)$ and with fixed transverse momentum $\left(p_{t}=0.3 \mathrm{GeV} / \mathrm{c}(\mathrm{a}), p_{t}=1.0 \mathrm{GeV} / \mathrm{c}(\mathrm{b})\right)$. In both plots the kaon sample was fitted with three different mass hypotheses: pion mass hypothesis (red), kaon mass hypothesis (blue) and proton mass hypothesis (green). For low momentum tracks, using the wrong mass hypothesis introduces a large bias in the momentum reconstruction. The effect is not as pronounced in case of high momentum tracks.

\section{Track Fitting}

The unique set of track candidates passes through the track fitting package GENFIT2 [10]. This package is a more general and extended version of its predecessor GENFIT [11], a toolkit originally developed for the PANDA collaboration [12] framework. GENFIT2 is an experimentindependent track fitting software. It provides track representations, track-fitting algorithms and graphic visualization. Currently, among the different track fitting algorithms implemented in the GENFIT2 package, the Belle II framework uses a Deterministic Annealing Filter (DAF). The DAF helps to reject outlying measurements and to downweight distant hits. Thanks to its adaptability, the package allows an easy treatment of hits coming from different subdetectors, as well as the presence of a not-uniform magnetic field and of an energy loss dependent on the particle type.

One of the features implemented in Belle II is the usage of the track fitting with three different mass hypotheses in parallel (namely pion, kaon and proton mass hypothesis). At low momentum, the usage of a wrong mass hypothesis can cause a large bias in the track momentum, while the effect is not as severe when considering high momentum tracks. This is shown in Fig. 3.

\section{Vertexing}

There are three different vertex fitters implemented in the Belle II framework: KFit, RAVE and TreeFitter.

KFit was developed for the previous experiment and it is based on a least square minimization approach. One of the main features of this fitter is the possibility to fit channels with neutral tracks, under the assumption that they originate from the interaction point $(0,0,0)$. 


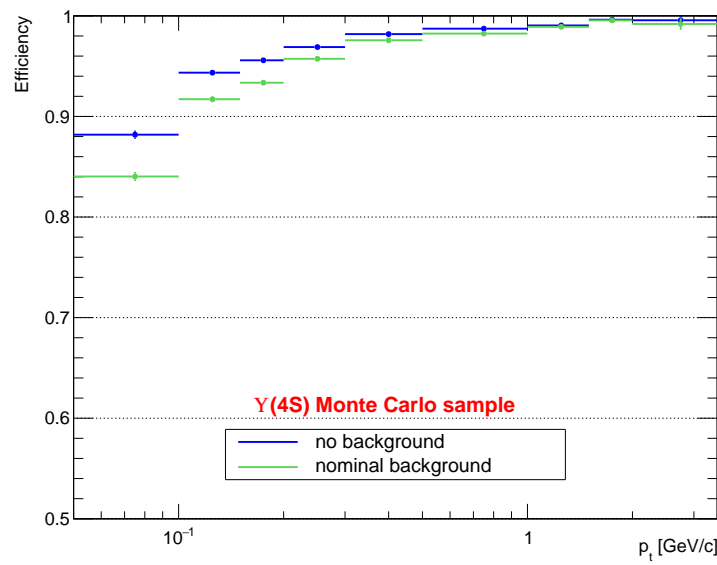

(a) Track finding efficiency as a function of the transverse momentum of the track candidate $p_{t}$

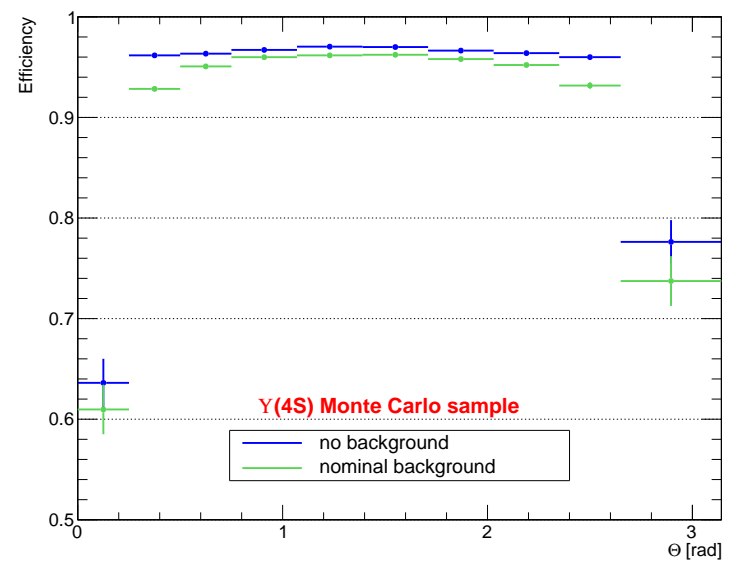

(b) Track finding efficiency as a function of the polar angle of the track candidate $\theta$

Figure 4: Belle II Track finding efficiency computed using a Monte Carlo simulation. The plots were obtained generating $10000 \Upsilon(4 S)$ generic events with the EvtGen package [1], simulated without (blue points) and with (green points) nominal background.

RAVE [13] is a detector-independent toolkit, originally developed for the CMS [14] reconstruction software. It is based on a generalization of the Kalman Filter, downweighting the contribution of outlier tracks in case of multiple tracks fitting. Contrary to KFit, RAVE can not handle and fit channels with neutral tracks.

TreeFitter [15] is a relatively new toolkit implemented in the Belle II framework. Compared to the previous two vertex fitters described, it uses a completely different approach in fitting particle decay trees, namely a global one instead of the canonical leaf-by-leaf one. This technique was first suggested and implemented in the BaBar experiment [16]. It uses a Kalman Filter approach to globally fit decay chains, without fitting each vertex at a time. In the algorithm different types of constraints are implemented, making it suitable for various purposes. Being (as already stated) a global fitting technique, it is particularly suitable for background rejection in decay trees containing neutral particles, an important feature for the Belle II physics program.

\section{Performance}

The Belle II tracking and vertexing algorithms have been widely tested using Monte Carlo simulations. The track finding efficiency, defined as the number of track candidates associated to a Monte Carlo particle divided by the total number of Monte Carlo particles generated inside the detector acceptance, can be computed simulating generic $\Upsilon(4 S)$ events; it is $\sim 96.5 \%$ when the background is not included in the simulation and $\sim 95.2 \%$ when it is included. In the future, the background simulation may change, in order to better describe what we see in data. In Fig. 4 the track finding efficiency is shown as a function of the transverse momentum $p_{t}$ (a) and as a function of the polar angle $\theta(b)$. Very low momentum tracks have a lower track finding efficiency since they do not reach the drift chamber and have therefore a smaller number of hits. Furthermore, the drop in the efficiency both in the forward and backward region of the detector is due to geometrical 


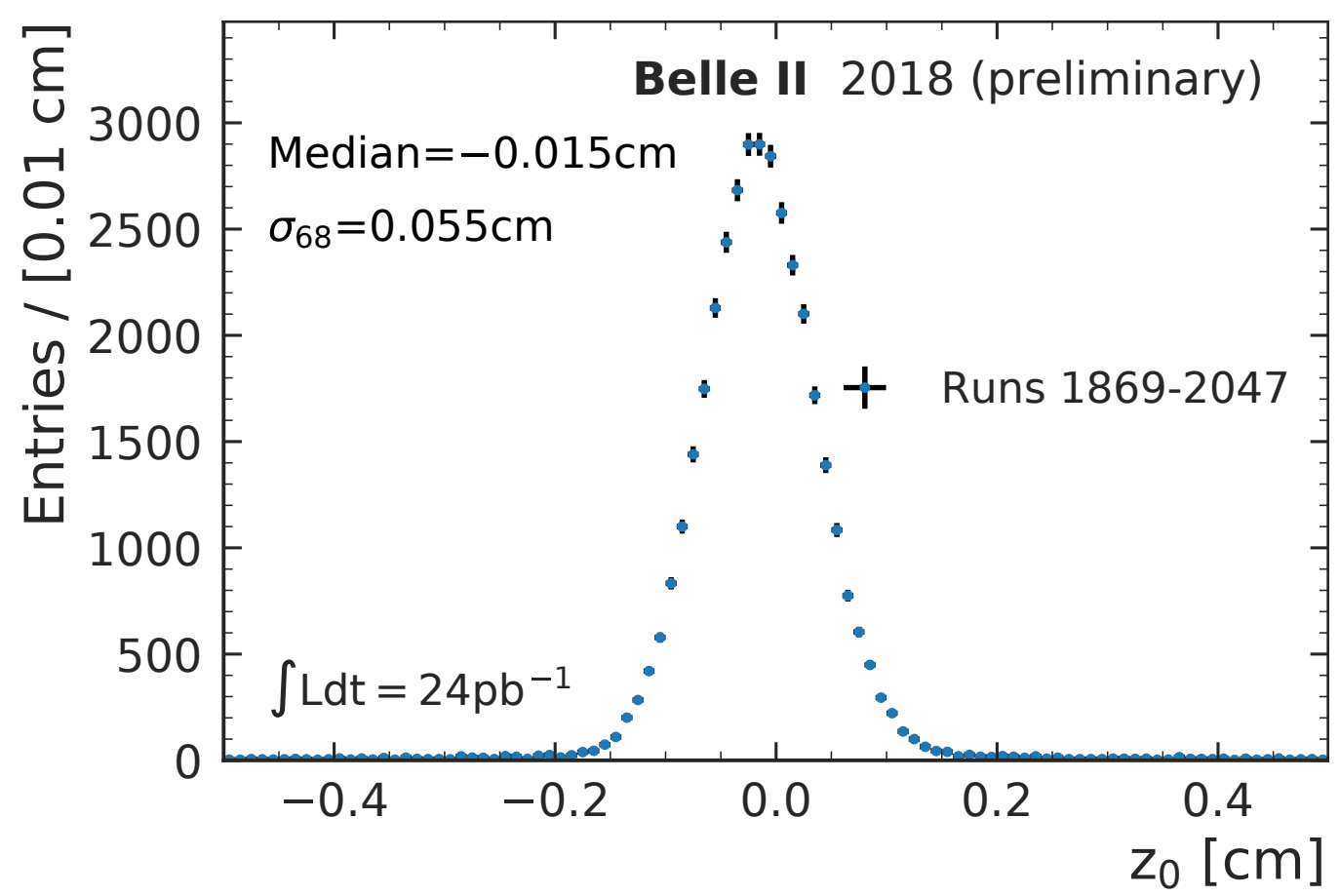

Figure 5: Distribution of the longitudinal component of the interaction vertex estimated using $z_{0}$ parameter of single tracks originating from the interaction vertex [18].

acceptance.

In April 2018, first data was collected during the machine commissioning phase, which represented the first opportunity to test the framework on real data. Thanks to the very good shape of the software, the data collection and the first physics signals and plots went almost hand in hand. Among the first important results was the measurement of the effective bunch length, using tracks passing the sector equipped with the PXD and SVD sensors. The large crossing angle of the beams in the nano-beam scheme leads to a narrow width of the collision points distribution in the $z$ direction. It is expected to be around $0.5 \mathrm{~mm}$, compared to $1 \mathrm{~cm}$ at Belle. This is confirmed in data, see Fig. 5.

\section{Outlook}

After a phase devoted to the commissioning of the detector, the Belle II experiment is ready to start the data taking and join the squad of particle physics experiment in the search for new physics. The tracking of Belle II detector has been tested thoroughly using both Monte Carlo simulation and the first collision data, and its performance found to be sufficient for the experiment's needs.

\section{Acknowledgment}

The author would like to thank the organizers of the $27^{\text {th }}$ International Workshop on Vertex Detectors (VERTEX2018) for the invitation to give the talk and the colleagues for the stimulating discussions, their support and their various contributions. This work was supported by the 
Bundesministerium für Bildung und Forschung under Grant No. 05H15UMKBA and a research Grant by the Johannes Gutenberg-Universität Mainz, Germany.

\section{References}

[1] D. J. Lange, The EvtGen particle decay simulation package, Nucl. Instrum. Meth. A, 462, (2001), 152, [doi:10.1016/s0168-9002(01)00089-4].

[2] E. Kou, P. Urquijo et al., The Belle II Physics Book, (2018), [arXiv: 1808 . 10567].

[3] Belle II official Website, [Online] Available: https: / / www . belle2.org/archives [February $\left.11^{\text {th }}, 2019\right]$, High Energy Accelerator Research Organization (KEK), Tsukuba, Japan.

[4] T. Abe et al, Belle II Technical Design Report (2010), [arXiv: 1011 . 0352].

[5] T. Keck, FastBDT: A Speed-Optimized Multivariate Classification Algorithm for the Belle II Experiment, Computing and Software for Big Science, 1, (2017), 2, [https://doi.org/10.1007/s41781-017-0002-8].

[6] V. Trusov, Development of Pattern Recognition Algorithms for the central Drift Chamber of the Belle II Detector, PhD Thesis, Karlsruhe Institute of Technology, (2016), [IEKP-KA/2016-31].

[7] J. Lettenbichler, Real-Time Pattern Recognition in the Central Tracking Detector of the Belle II Experiment, PhD Thesis, Technische Univertität Wien, (2016).

[8] J. Wagner, Track Finding with the Silicon Strip Detector of the Belle II Experiment, Master thesis, Karlsruhe Institute of Technology, (2017), [ETP-KA/2017-28].

[9] V. Bertacchi, Development and performance of the track finder for the Belle II Vertex Detector, Master Thesis, Università di Pisa, (2016-2017).

[10] T. Bilka et al., Implementation of GENFIT2 as an experiment independent track-fitting framework, (2019), [arXiv:1902.04405v2].

[11] J. Rauch and T. Schlüter, GENFIT, a Generic Track-Fitting Toolkit, (2016), [arXiv:1410.3698v2].

[12] PANDA experiment Website, [Online] Available: https : / / panda.gsi.de/ [February 13 ${ }^{\text {th }}$, 2019], Facility for Antiproton and Ion Research (FAIR), GSI Helmholtzzentrum für Schwerionenforschung, Darmstadt, Germany.

[13] W. Waltenberger, W. Mitaroff, F. Moser, RAVE - a detector-independent toolkit, Nucl. Instrum. Methods Phys. Res. A, 581, (2007), 549, [10.1016/j.nima.2007.08.048].

[14] CMS experiment website, [Online] Available: https : / / cms . cern / [February 13 ${ }^{\text {th }}, 2019$ ], Conseil Européen pour la Recherche Nucléaire (CERN), Geneva, Switzerland.

[15] J. F. Krohn et al., Global Decay Chain Vertex Fitting at B-Factories, (2019), [arXiv:1901.11198].

[16] W. D. Hulsbergen, Decay Chain Fitting with a Kalman Filter, Nucl. Instrum. Meth. A, 552, (2005), 566, [arXiv:physics/0503191].

[17] M. Bona et al., SuperB: A High-Luminosity Asymmetric e+e-Super Flavor Factory. Conceptual Design Report, (2007), [arXiv:0709.0451].

[18] N. Braun et al., Study of the collision point properties, (2018), [BELLE2-NOTE-PL-2018-0 08]. 